\title{
Randomized, controlled trial of laser vs. bipolar plasma vaporization treatment of benign prostatic hyperplasia
}

\author{
Thomas A.A. Skinner, MSc, MD; Robert J. Leslie, MSc, MD; Stephen S. Steele, MD, FRCSC; \\ J. Curtis Nickel, MD, FRCSC
}

Department of Urology, Queen's University, Kingston, ON, Canada

Cite as: Can Urol Assoc J 2017;11 (6):194-8. http://dx.doi.org/10.5489/cuaj.4213

\section{Abstract}

Introduction: Prostate vaporization technology is becoming a standard of care for treatment of moderate, symptomatic benign prostatic hyperplasia (BPH). We compared two transurethral prostate vaporization technologies with respect to cost, efficiency, efficacy, safety, and surgical team satisfaction.

Methods: Fifty-five patients meeting standardized symptom criteria for $\mathrm{BPH}$ were randomized to either Olympus Plasma Button ${ }^{\mathrm{TM}}$ or Biolitec EVOLVE ${ }^{\circledR}$ diode laser vaporization. Primary outcome of cost with secondary outcomes of clinical efficacy, resection time, surgical team satisfaction, and safety were analyzed. Followup was carried out at six and 12 weeks. Patient factors included baseline, as well as six- and 12-week International Prostate Symptom Score (IPSS) with quality of life (QoL) scores. We recorded surgical team satisfaction with a Likert-style survey investigating ease of set-up, reliability, efficiency, and ability to reach desired endpoint. All complications or side effects detected within three months and the resulting management were included in the cost analysis.

Results: Mean cost per patient was $\$ 3418$ for the Olympus group and $\$ 4564$ for Biolitec $(p<0.05)$. Surgical vaporization time was significantly less for the Olympus group, 24.3 vs. 33.5 minutes $(p<0.05)$. Surgical and nursing staff preferred the Olympus device $(p<0.05)$. IPPS symptom improvement and complication rates were similar between groups. Patients in the Biolitec arm had more intraoperative bleeding episodes requiring conversion to monopolar transurethral resection of the prostate (TURP) (three vs. none). Conclusions: In a head-to-head randomized trial, Olympus Plasma Button transurethral vaporization was more cost-effective, faster, and preferred by surgical staff when compared to Biolitetec Diode Laser vaporization. Both devices showed similar safety and efficacy.

\section{Introduction}

Benign prostatic hyperplasia (BPH) affects 50\% of men over age 50 and progresses to bothersome lower urinary tract symptoms (LUTS) in up to one-third of men. ${ }^{1-4}$ For eight dec- ades, the gold standard surgery for bothersome benign prostatic obstruction (BPO) has been monopolar transurethral resection of the prostate (TURP). ${ }^{5}$ Multiple minimally invasive techniques have emerged in the past two decades, with laser and electro-vaporization becoming a new standard. ${ }^{6}$ Transurethral vaporization of the prostate (TUVP) provides better hemostasis and allows for the use of isotonic irrigation fluid. This permits better intraoperative visualization, treatment of those at increased risk of bleeding, and has been shown to result in shorter hospital stays, shorter postoperative catheterization, and less electrolyte derangements (TUR syndrome). ${ }^{7}$ Despite these advantages, many Canadian institutions have been slow to adopt a prostate vaporization program. In an effort to inform our own and other Canadian institutions, we set out to compare two TUVP platforms being considered for use in our institution in a prospective, randomized fashion: the Olympus Plasma Button ${ }^{\mathrm{TM}}$ and the Biolitec EVOLVE ${ }^{\circledR}$ DUAL wavelength diode laser.

Lasers (light amplification by stimulated emission of radiation) have been in the armamentarium of urologists for decades, but only recently for the management of $\mathrm{BPH}$. While long-term data for diode lasers is scarce, the available evidence suggests they provide results comparable to TURP with the advantages seen with other vaporization platforms. ${ }^{7}$ The primary challenge with diode lasers is deep tissue penetration and coagulative necrosis. ${ }^{7}$ This is associated with dysuria, passage of sloughed tissue, and higher reoperation rates for bladder neck stenosis. ${ }^{8}$ The Biolitec EVOLVE is the first dual-wavelength device available for transurethral prostate surgery. It produces wavelengths of $980 \mathrm{~nm}$ and $1470 \mathrm{~nm}$, designed to combine excellent coagulation with tissue ablation.

Bipolar TURP (B-TURP) allows for treatment in normal saline irrigation, preventing TUR syndrome. While B-TURP is associated with less bleeding, previous devices have failed to catch on as a replacement for TURP due to limited evidence of superiority. ${ }^{9}$ Recently, an evolution of B-TURP, bipolar plasma vaporization of the prostate, has emerged. 
This allows efficient tissue vaporization while maintaining all the benefits of bipolar energy. The Olympus Plasma Button has demonstrated excellent results in tissue vaporization with significantly less bleeding than TURP and boasts reduced hospital stays and catheterization time. ${ }^{10}$

In the current economic climate, adopting new technology is not always feasible. Surgical management of BPH is common in Canada, with 20000 TUR procedures performed annually. ${ }^{11}$ With an average cost of $\$ 3447$ per TURP in Canada, even a modest reduction in cost would have substantial financial implications. ${ }^{12}$ While only $7.6 \%$ of TUR procedures in 2011 employed minimally invasive techniques, this number doubled from 2007-2011. ${ }^{11}$ If this trend continues, we could see TUVP overtake TURP in the next decade. A few groups have shown vaporization to be more cost-effective than TURP, but to date no one has performed a cost comparison of these devices in Canada. ${ }^{13-15}$ Here, we compared cost, clinical outcomes, safety, and surgical team preference of two transurethral prostate vaporization systems using the Biolitec EVOLVE DUAL diode laser and the Olympus Plasma Button in a prospective, randomized trial.

\section{Methods}

This prospective, randomized, single-blinded study was conducted from July 2014 to June 2016 at a tertiary care centre in Ontario, Canada. Ethics approval was obtained from our institution. Patients with moderate to severe, symptomatic $\mathrm{BPH}$ who consented to participate in this trial were randomized to either the Olympus or Biolitec device. Patients were required to meet the following inclusion criteria: age over 45, International Prostate Symptom Score (IPSS) $\geq 12$, estimated prostate volume on digital rectal exam (DRE) $\geq 30$ cc (as this is a real-life clinical practice study, prostate size and post-void residual were not mandatory). Anticoagulation was held for all procedures. Individuals with prior invasive intervention for $\mathrm{BPH}$, prostate-specific antigen (PSA) level $>10 \mathrm{ng} / \mathrm{ml}$, urinary retention, medical condition unfit for surgery, history of prostate cancer, documented prostatitis within the past three months, known bleeding disorder, unable to follow directions or sign informed consent due to organic brain or psychiatric disease, or those with history of substance abuse, which would affect compliance, were excluded from the study.

Sample size determination was undertaken using Minitab statistical software. ${ }^{16}$ Power was calculated to detect a cost difference of $\$ 800$. To detect this difference at $90 \%$ power, 25 patients were required per group $(\alpha=0.05)$. We planned to randomize 60 patients over a two-year period.

Patients were evaluated with an initial screening appointment to assess candidacy. Those interested in participating received verbal and written instructions and completed the informed consent agreement. They again participated in the consent process on the day of surgery. Prior to their operation, demographic information was collected and participants completed an IPSS questionnaire. Patients were randomized into two groups using GraphPad QuickCalcs, random number generator software. ${ }^{17}$ The surgical team was notified of the patient's randomization status before the procedure.

Mentorship training on both systems was followed by operating on two patients per surgeon on each device before eligible patients were randomized to either the Olympus or Biolitec procedure and performed by one of two surgeons (JCN or SSS). The lead surgeon and scrub nurse were asked to complete a survey rating their experience following each case. A Likert-style scale was used to rate the following categories from 1-5: Ease of set-up, reliability of equipment, efficiency of resection, ability to reach desired endpoint, and overall rating, for a total out of 25 . All patients discharged the day of surgery had an average of one hour of irrigation, while those admitted had overnight irrigation.

Followup appointments were scheduled at six weeks and three months postoperatively, with IPSS repeated at these visits. All ancillary visits and treatments for side effects or complications (related to the initial procedure) diagnosed within the first three months were included in the cost analysis, even when these took place beyond the threemonth window. Cost projections were derived from multiple sources. Procedure and followup visit costs came from our own institution's cost for care of non-insured Canadians and correlated with Canadian Institute for Health Information (CIHI) data. ${ }^{12}$ Equipment and disposable costs were provided from the respective companies. Medication prices were derived from the Ontario Drug Benefit (ODB) database. ${ }^{18}$

Intention-to-treat analysis was performed. For baseline demographics, IPSS, quality of life (QoL) scores, cost, resection time, and nursing and surgeon satisfaction data means from each group were compared with two-tailed MannWhitney $U$ test for non-parametric data, with a significance value of 0.05 . Cost outliers were determined as those that fell beyond two standard deviations (SD) of the mean.

\section{Results}

Enrollment was terminated at 55 patients when the two-year window was reached (30 Olympus, 25 Biolitec). Preoperative patient characteristics (age, prostate volume, IPSS, and QoL scores) were equivalent between groups (Table 1). Mean IPSS and QoL scores at six-week followup showed similar improvement between groups, with IPSS scores improving by 12 in the Olympus group and 11 in the Biolitec group $(p=0.60)$. At three months, mean IPSS scores in the Olympus arm and Biolitec arm improved to 9.9 and 9.4, respectively $(p=0.62)$, an improvement of 12.7 and 11.1 , respectively. Mean QoL scores were similarly improved to 1.65 in both 
Skinner et al.

\begin{tabular}{|c|c|c|c|}
\hline & Olympus & Biolitec & p \\
\hline Number of patients & 30 & 25 & \\
\hline Mean age at entry & 71.8 & 69.4 & 0.41 \\
\hline Mean prostate volume & 47.8 & 46.6 & 0.92 \\
\hline Mean prostate-specific antigen & 2.3 & 1.4 & 0.32 \\
\hline Median lobe presence & $40 \%$ & $36 \%$ & 0.76 \\
\hline IPSS at entry & 22.6 & 20.5 & 0.12 \\
\hline Quality of life at entry & 4.7 & 5.1 & 0.16 \\
\hline
\end{tabular}

arms ( $p=0.90)$ from 4.7 and 5.1 for Olympus and Biolitec, respectively (Fig. 1).

Mean cost per patient was $\$ 3418$ for the Olympus group and $\$ 4564$ for Biolitec treatment $(\mathrm{p}<0.05)$ (Fig. 2A). With outliers excluded, these costs improved to $\$ 2946$ and $\$ 3913$ for Olympus and Biolitec, respectively $(p<0.05)$. Surgical resection time was significantly less for the Olympus group, 24.3 vs. 33.5 minutes $(p<0.05)$ (Fig. 2B). Surgical and nursing staff preferred using the Olympus device over the Biolitec device with total scores 23.4 vs. 16.7 for surgeons and 24.2 vs. 17.9 for nursing staff (out of 25), respectively $(p<0.05)$ (Fig. 2C). Postoperative admission (and same-day discharge) rates were similar between groups, with the majority of admissions noted during the early learning curve (Table 2). Mean length of catheterization time was 2.1 days for Olympus and 2.2 days for Biolitec $(p=0.15$; a single outlier with 55 days of indwelling catheter was excluded from the Biolitec group).

Safety and complication rates were similar between groups (Table 3), with the exception of significant intraoperative bleeding episodes. Patients in the Biolitec arm had more intraoperative bleeding episodes requiring conversion to monopolar TURP (three events vs. none for Olympus). Overall, both devices were safe; there were no Grade IV or $\mathrm{V}$ adverse events in either group.

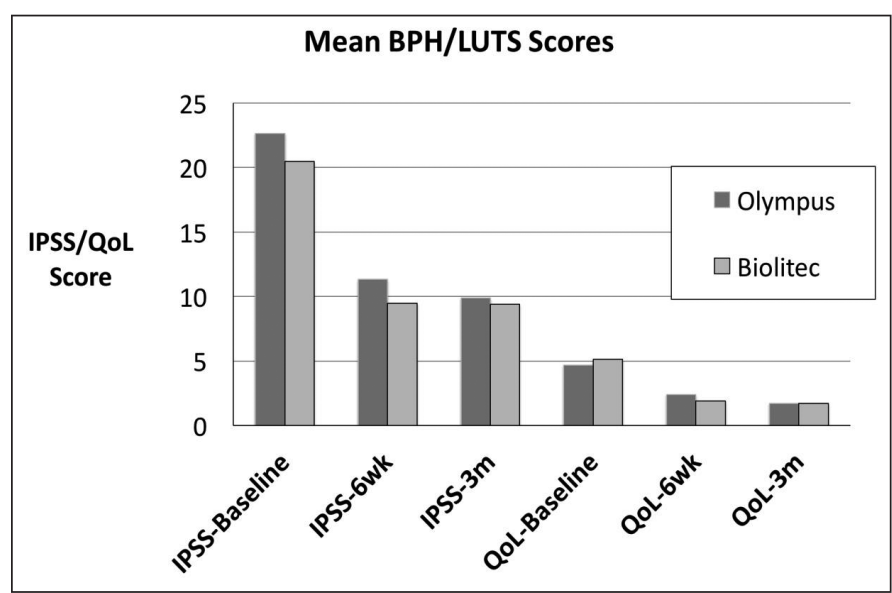

Fig. 1. International Prostate Symptom Score (IPSS) and Quality of Life (QoL) scores over time. BPH: benign prostatic hyperplasia; LUTS: lower urinary tract symptoms.

\section{Discussion}

This is the first cost analysis comparing two TUVP devices in a Canadian centre. This study was designed to be a reallife clinical practice comparison of the two devices being considered for use in our institution. Other devices initially considered were deemed ineligible because of either nonavailability in Canada or refusal to agree to a head-to-head comparison. The results of our study are much stronger than those generated by an anecdotal experience of few cases undertaken on a device loaned for a trial period. Our data suggests that the Olympus Plasma Button is more cost effective, faster, and preferable to use within our centre, when compared to the Biolitec EVOLVE diode laser. Both devices were equivalent with respect to patient satisfaction, voiding outcomes, and safety.

The cost difference between groups was multifactorial. Consumables (laser fiber vs. button electrode) cost $\$ 307$ more per case in the laser group. Additional physician visits, hospi-

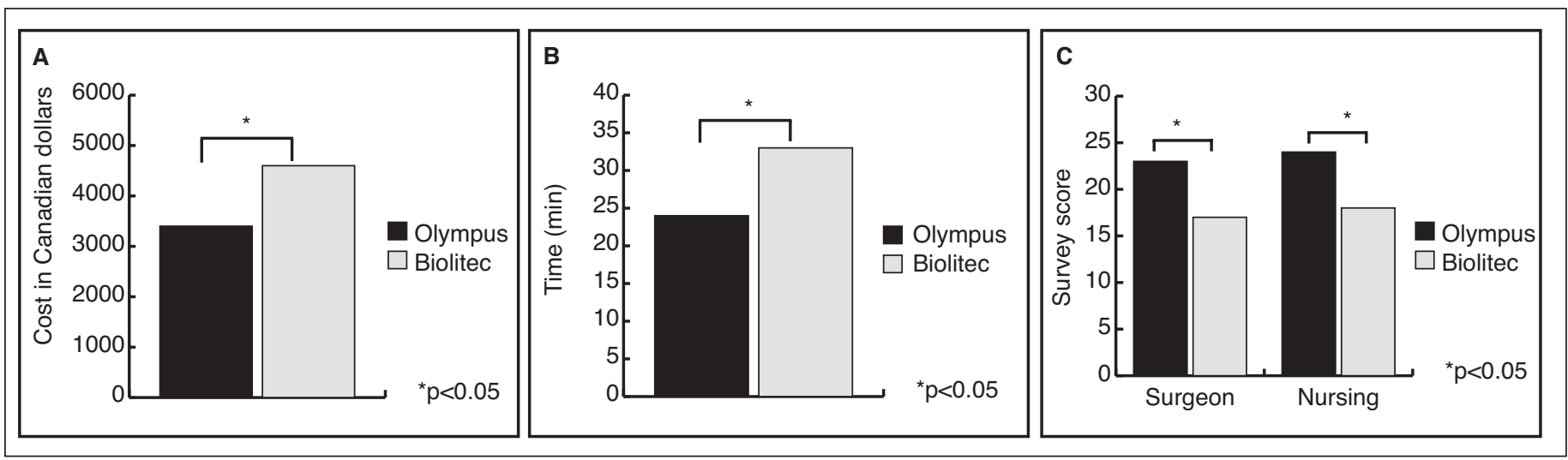

Fig. 2. (A) Mean cost per patient in Canadian dollars; (B) mean vaporization time required per patient; and (C) mean surgeon and nursing satisfaction score (out of a total of 25 points). 


\begin{tabular}{|c|c|c|c|c|c|}
\hline & \multicolumn{2}{|c|}{ Olympus } & \multicolumn{2}{|c|}{ Biolitec } & \multirow[b]{2}{*}{$\mathbf{p}$} \\
\hline & $\mathbf{n}$ & $\%$ & $\mathbf{n}$ & $\%$ & \\
\hline Early (1-15) & $8 / 12$ & 67 & $2 / 3$ & 67 & 1 \\
\hline Late (16-55) & $5 / 18$ & 20 & $6 / 22$ & 27 & 0.97 \\
\hline Overall & $12 / 30$ & 40 & $8 / 25$ & 32 & 0.54 \\
\hline
\end{tabular}

talizations, and procedures provided the majority of the costs for both groups. A few individuals consumed the bulk of these costs; however, when outliers were excluded, the difference between groups still remained nearly $\$ 1000$. There is conflicting data regarding whether vaporization procedures are less expensive than TURP, but TUVP probably costs less. ${ }^{13-15,19}$ When compared to the national average TURP cost reported by $\mathrm{CIHI}(\$ 3447)$, the cost of Olympus TUVP was comparable (\$3418). ${ }^{12}$ With outliers removed, this device averaged $\$ 2946$ per patient, a savings of $\$ 500$ per treatment over the current cost of TURP. This represents a potential savings of $\$ 10 \mathrm{mil}$ lion per annum over 20000 procedures. Care must be taken when interpreting these results, however, as $\mathrm{ClHI}$ data likely measures some different cost factors. Our costs include a significant number of admissions, as per early trial protocol. After our initial learning curve of 15 patients, we routinely sent patients home with only $20-27 \%$ of patients requiring admission. Performing TUVP routinely as day surgery would increase the cost savings of TUVP.

With respect to resection time, we speculate that learning curve plays a role in the difference between groups. The Olympus device resembled the resectoscope already in use at our institution; however, when resection time was broken down by tertile (data not shown), there was no significant improvement in time between tertiles in the laser group. This may be partly related to the TURP conversions that added considerable surgical time. Other studies ${ }^{20}$ have commented that the learning curve for this device is short; however, surgeons at our institution felt that the laser procedure was less intuitive. It is also likely with our small sample size shared by two surgeons that some improvement was still to be expected, as Gross et al demonstrated a learning curve of over 200 cases with another laser device. ${ }^{21}$ Surgical time is an important consideration in Canada, as surgeons have limited access to operating room time and may be able to better manage wait lists by completing more TUVPs in a given day.

Patient outcomes (IPSS score), patient satisfaction (QoL score), and safety were not significantly different between groups. Both groups showed over 10-point reduction in average IPSS scores after treatment, and QoL scores decreased by an average of over three following treatment. This correlates with the symptomatic improvement seen in other TUVP studies. ${ }^{10,22}$ Safety profiles were also comparable. While three individuals in the laser group required conversion to TURP for hemostasis, none these individuals required blood transfusions and no adverse events occurred postoperatively related to bleeding. Other minimally invasive laser technologies have documented conversions, with $21.6 \%$ of Green Light Laser procedures requiring conversion to TURP in one study. ${ }^{23}$ We should also note that the majority of patients in both arms were discharged home the day of surgery. This is an important consideration over TURP, as overnight beds are an increasingly scarce resource in Canadian hospitals.

TUVP is a safe, effective, and potentially cost-saving approach to management of BPO in a Canadian centre. We should continue to strive to find cost-effective technologies to improve Canadian healthcare. These findings support the adoption of minimally invasive devices by Canadian institutions and may help inform other Canadian institutions deciding on device selection.

\section{Conclusion}

The Olympus Plasma Button was more cost-effective and surgically efficient for management of moderate, symptom-

\begin{tabular}{|c|c|c|c|c|c|c|c|c|}
\hline \multirow{2}{*}{$\begin{array}{l}\text { Adverse event } \\
\text { Clavien-Dindo }\end{array}$} & \multicolumn{4}{|c|}{ Olympus } & \multicolumn{4}{|c|}{ Biolitec } \\
\hline & $\mathbf{I}$ & II & Illa & IIIlb & $\mathbf{I}$ & II & IIla & IIllb \\
\hline Convert to TURP & 0 & 0 & 0 & 0 & $3(12 \%)$ & 0 & 0 & 0 \\
\hline Bladder injury & 0 & 0 & 0 & 0 & 0 & 0 & $1(4 \%)$ & 0 \\
\hline Clinically significant storage LUTS & 0 & $(17 \%)$ & 0 & 0 & 0 & $6(24 \%)$ & 0 & 0 \\
\hline ER/GP visits & 0 & $11(37 \%)$ & 0 & 0 & 0 & $13(52 \%)$ & 0 & 0 \\
\hline Clot retention & 0 & $4(13 \%)$ & 0 & 0 & 0 & $3(12 \%)$ & 0 & 0 \\
\hline Readmission & 0 & $3(10 \%)$ & 0 & 0 & 0 & $3(12 \%)$ & 0 & 0 \\
\hline Additional urology visits & 0 & $8(27 \%)$ & 0 & 0 & 0 & $7(28 \%)$ & 0 & 0 \\
\hline Postoperative cystoscopy & 0 & 0 & $6(20 \%)$ & 0 & 0 & 0 & $5(20 \%)$ & 0 \\
\hline Urethral stricture & 0 & 0 & $2(7 \%)$ & 0 & 0 & 0 & $2(8 \%)$ & 0 \\
\hline Re-TURP & 0 & 0 & & $1(13 \%)$ & 0 & 0 & 0 & $1(4 \%)$ \\
\hline
\end{tabular}

ER: emergency room; GP: general physician; LUTS: lower urinary tract symptoms; TURP: transurethral resection of the prostate. 
atic BPH compared to the Biolitec Evolve diode laser, however, both devices proved to be effective and safe.

Competing interests: Dr. Steele has been an advisor for Allergan and Astellas; a speaker for Abbott and Astellas; has received grants from Astellas and Pfizer; and has participated in clinical trials supported by Astellas and Pfizer. The remaining authors report no competing personal or financial interests.

Acknowledgements: This study was supported by the Ontario Academic Health Centres - Alternate Funding Plan Innovation Fund. The equipment was provided by Olympus and Biolitec. We thank Dr. Amir Rumman, who assisted the research team in developing the analytical models.

This paper has been peer-reviewed.

\section{References}

1. Ziada A, Rosenblum M, Crawford ED. Benign prostatic hyperplasia: An overview. Urology 1999:53:1-6. https://doi.org/10.1016/S0090-4295(98)00532-9

2. Roehrborn CG. Benign prostatic hyperplasia: An overview. Rev Urol 2005;7:S3-14.

3. Kapoor A. Benign prostatic hyperplasia (BPH) management in the primary care setting. Can I Urol 2012;19:10-7.

4. Madersbacher S, Alivizatos G, Nordling J, et al. EAU 2004 guidelines on assessment, therapy and followup of men with lower urinary tract symptoms suggestive of benign prostatic obstruction (BPH guidelines). Eur Urol 2004;46:547-54. https://doi.org/10.1016/i.eururo.2004.07.016

5. Nickel JC, Méndez-Probst CE, Whelan TF, et al. 2010 Update: Guidelines for the management of benign prostatic hyperplasia. Can Urol Assoc J 2010;4:310-6. https://doi.org/10.5489/cuaj.10124

6. Bachmann A, Woo HH, Wyler S. Laser prostatectomy of lower urinary tract symptoms due to benign prostate enlargement: A critical review of evidence. Curr Opin Urol 2012; 22:22-33. https://doi.org/10.1097/MOU.0b013e32834dd0ed

7. Rieken $M$, Bachmann $A$. Laser treatment of benign prostate enlargement — which laser for which prostate? Nat Rev Urol 2014;11:142-52. https://doi.org/10.1038/nrurol.2014.23

8. Shaker HS, Shoeb MS, Yassin MM, et al. Quartz head contact laser fiber: A novel fiber for lase ablation of the prostate using the $980 \mathrm{~nm}$ high-power diode laser. J Urol 2012;187:575-9. https://doi.org/10.1016/i.juro.2011.09.153

9. Hueber PA, Al-Asker A, Zorn KC. Monopolar vs. bipolar TURP: Assessing their clinical advantages. Can Urol Assoc J 2011;5:390. https://doi.org/10.5489/cuaj.11263
10. Geavlete B, Georgescu D, Multescu R, et al. Bipolar plasma vaporization vs. monopolar and bipolar TURPa prospective, randomized, long-term comparison. Urology 2011;78:930-5. https://doi.org/10.1016/i. urology.2011.03.072

11. Hueber PA, Zorn K. Canadian trend in surgical management of benign prostatic hyperplasia (BPH) and laser therapy from 2007-2008 to 2011-2012. Can Urol Assoc J 2013;7:582-6. https://doi.org/10.5489/cuai.203

12. Canadian Institute for Health Information. Available at http://www.cihi.ca. Accessed July 2016.

13. Liatsikos E, Kyriazis I, Kallidonis $\mathrm{P}$, et al. Photoselective Greenlight ${ }^{\mathrm{TM}}$ laser vaporization vs. transurethral resection of the prostate in Greece: A comparative cost analysis. J Endourol 2012;26:168-73. hitps://doi.org/10.1089/end.2011.0089

14. van Melick HH, van Venrooij GE, van Swol CF, et al. Cost aspects of transurethral resection of the prostate, contact laser prostatectomy, and electrovaporization. Urology 2004;63:882-6. https://doi.org/10.1016/i.urology.2003.12.008

15. Fraundorfer MR, Gilling PJ, Kennett KM, et al. Holmium laser resection of the prostate is more cost effective than transurethral resection of the prostate: Results of a randomized, prospective study. Urology 2001;57:454-8. https://doi.org/10.1016/S0090-4295(00)00987-0

16. Minitab Statistical Software. [Computer Software]. v 15.1, 2006, LEAD Technologies, Philadelphia, PA (www.minitab.com).

17. GraphPad.com, SanDiego: QuickCalcs @2016GraphPad Software, Inc. (http://graphpad.com/quickcalcs/randomizel). Accessed Sept 19, 2016

18. Ontario Drug Benefit Formulary/Comparative Drug Index. Ontario: Drug Programs Policy and Strategy Branch Ontario Public Drug Program Ministry of Long-Term Care. Edition 42. Effective April 27, 2017. Avallable at https://www.formulary.health.gov.on.ca/formulary/. Accessed May 18, 2017.

19. Whitty JA, Crosland P, Hewson K, et al. A cost-minimization analysis comparing photoselective vaporisation (PVP) and transurethral resection of the prostate (TURP) for the management of symptomatic benign prostatic hyperplasia (BPH) in Queensland, Australia. BJU Int 2014;113:21-8. https://doi.org/10.1111/bju.12051

20. Muir G, Gomez F, Choi B, et al. Multicentre prospective study of the Evolve: Dual $150 \mathrm{~W}$ laser for bladder oufflow obstruction: Preliminary results. Urology 2011;78:S59.

21. Gross AJ, Netsch C, Knipper S, et al. Complications and early postoperative outcome in 1080 patients after thulium vapoenucleation of the prostate: Results at a single institution. Eur Urol 2013;63:859-67. https://doi.org/10.1016/i.eururo.2012.11.048

22. Reich 0 , Schlenker $B$, Gratzke $C$, et al. Plasma vaporisation of the prostate: Initial clinical results. Eur Urol 2010;57:693-8. https://doi.org/10.1016/i.eururo.2009.05.031

23. Elmansy $H$, Baazeem $A$, Kotb $A$, et al. Holmium laser enucleation vs. photoselective vaporization for prostatic adenoma greater than $60 \mathrm{ml}$ : Preliminary results of a prospective, randomized clinical trial. J Urol 2012;188:216-21. https://doi.org/10.1016/i.juro.2012.02.2576

Correspondence: Dr. J. Curtis Nickel, Department of Urology, Queen's University, Kingston, ON, Canada; jin@queensu.ca 